\title{
Status and prospects of measurements of exclusive and diffractive processes with the ATLAS detector
}

\author{
Andrew Foster, on behalf of the ATLAS Collaboration* \\ University of Birmingham (UK) \\ E-mail: a.foster@cern.ch
}

Fiducial and differential cross-sections are presented for the exclusive production of pairs of leptons and $W$ bosons, measured across a range of centre-of-mass energies by the ATLAS Collaboration at the LHC. All measurements are compatible with Standard Model predictions. A measurement of the dijet production cross-section is presented differentially in variables which distinguish between diffractive and non-diffractive scattering. The first analysis utilising the new AFP detectors at ATLAS is detailed.

XXVI International Workshop on Deep-Inelastic Scattering and Related Subjects (DIS2018) 16-20 April 2018

Kobe, Japan

\footnotetext{
* Speaker.
} 


\section{Introduction}

An exclusive process is one in which the final state particles are all explicitly defined. At the LHC, this criterion is most feasible in events that do not result in dissociation of either of the protons. Accordingly, exclusive measurements are typically performed in the 'central' configuration, meaning a $t$-channel process, in which neither of the protons dissociate, resulting in a clean detector signal. The final state then comprises the two scattered, intact protons and a central system produced through interactions between the exchange particles from the protons. The exchange particles are most commonly strongly-interacting colour singlets, often dubbed the 'Pomeron', which is most easily conceptualised as a pair of colour-neutral gluons, although its exact constitution is not known [1]. It is also possible for these processes to be mediated by photons produced by the protons' electromagnetic fields, which under the equivalent photon approximation (EPA) can be treated as a quasi-real beam of photons with small virtuality, $Q^{2}<0.1 \mathrm{GeV}^{2}$ [2]. Such processes are identifiable through the exclusive final state with no other particles originating from the same primary vertex. Diffractive processes are similarly described by a $t$-channel colour singlet exchange in which at least one of the protons dissociates. These processes are typically identifiable through the presence of large regions in rapidity devoid of final state particles. Such a signature is exponentially suppressed in a typical non-diffractive hard interaction between partons in the incident protons. Both diffractive and central exclusive interactions can result in final states with protons at near-beam energy in the very forward region. The tagging of these scattered protons can aid in the identification of such events and improve upon current measurements. The analyses described in this article were performed on data collected by the ATLAS experiment [3] during proton-proton collisions at $\sqrt{s}=7,8$ and $13 \mathrm{TeV}$ provided by the LHC at CERN. The data used in the exclusive analyses presented were collected during 'nominal' running conditions while the diffraction analyses' data sets were collected during low pile-up runs ${ }^{1}$.

\section{Central Exclusive Measurements}

The exclusive dilepton, $\gamma \gamma \rightarrow l^{+} l^{-}$, production cross-section is well predicted within QED with an uncertainty below $2 \%$, associated with the elastic form factors [4]. At $\sqrt{s}=7 \mathrm{TeV}$, the exclusive di-electron and di-muon (Fig. 1a) cross-sections were measured, using an integrated luminosity of $4.6 \mathrm{fb}^{-1}$ [5]. Events were selected by requiring at least one collision vertex with two lepton candidates with $p_{T}>400 \mathrm{MeV}$ in the pseudorapidity, $\eta$, region $|\eta|<2.4$, triggered by single and double lepton triggers. An exclusivity requirement is applied on the track multiplicity and longitudinal isolation of the dilepton vertex, completely removing multijet, diboson and $t \bar{t}$ production backgrounds, leaving Drell-Yan, single (Fig. 1b) and double dissociation (Fig. 1c) as the only significant sources of background. To remove the majority of the Drell-Yan contribution, the Z-mass region is excluded in the dilepton invariant mass, vetoing on $70<m_{l^{+} l^{-}}<105 \mathrm{GeV}$. An additional selection is applied to the transverse momentum of the dilepton system of $p_{T}^{l^{+} l^{-}}<1.5 \mathrm{GeV}$, removing some of the proton-dissociative backgrounds. The final selection applied is a template fit to the acoplanarity $\left(1-\left|\Delta \phi_{l^{+} l^{-}}\right| / \pi\right)$ distribution to determine the scale factors for the exclusive and single dissociative Monte Carlo (MC) contribu-

\footnotetext{
${ }^{1}$ Pile-up, $\mu$, is defined as the mean number of interactions in a bunch crossing. During nominal running, $\mu \gg 1$.
} 


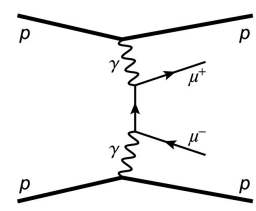

(a)

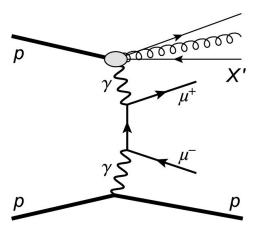

(b)

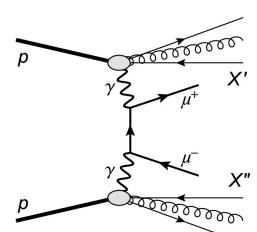

(c)



(d)

Figure 1: Schematic diagrams for (a) exclusive, (b) single dissociative and (c) double dissociative photon-initiated production of muon pairs in proton-proton collisions. The effect of additional interactions between the protons, referred to as absorptive effects, is shown in (d) [6].

tions. The generators used for the exclusive signal and single dissociative background are HERWIG++ 2.6.3 and LPAIR 4.0, respectively [7,8]. The scale factors for the exclusive di-electron and di-muon samples are measured to be $0.863 \pm 0.070$ (stat.) \pm 0.037 (syst.) \pm 0.015 (theor.) and $0.791 \pm 0.041$ (stat.) \pm 0.026 (syst.) \pm 0.013 (theor.). These factors correspond to a measurement of proton absorptive effects, where the final state is altered by additional interactions between the final state particles, predominantly strong interactions between the protons, see Fig. 1d. These factors are not present in the MC by default. Applying these correction factors multiplicatively to the predicted fiducial cross-section results in a measurement of the fiducial cross-section of $0.428 \pm 0.035$ (stat.) \pm 0.018 (syst.) pb and $0.628 \pm 0.032$ (stat.) \pm 0.021 (syst.) pb for the exclusive di-electron and di-muon processes respectively. These cross-section measurements are compared to the predictions accounting for the proton absorptive effects in Fig. 2a. The absorptive effects are calculated through the finite size approximation, which allows only photons outside of the proton radius, $r_{p}=0.64 \mathrm{fm}$, to initiate the two-photon interaction [9]. The corrected predictions are in agreement with the measured cross-section in both channels.

A measurement was also performed of the exclusive di-muon differential cross-section as a function of the di-muon invariant mass at $\sqrt{s}=13 \mathrm{TeV}$ [6]. The analysis selection is very similar to that used in the previously described analysis except only performed in the di-muon channel, using an altered Z-mass cut to only accept events with $m_{\mu^{+} \mu^{-}}<70 \mathrm{GeV}$. The integrated fiducial cross-section is measured to be $\sigma_{\gamma \gamma \rightarrow \mu^{+} \mu^{-}}^{\text {excl.fid. }}=3.12 \pm 0.07$ (stat.) \pm 0.14 (syst.) pb, which can be compared with the HERWIG prediction, corrected for absorptive effects, of $\sigma_{\gamma \gamma \rightarrow \mu^{+} \mu^{-}}^{\mathrm{EPA}, \mathrm{cor} .}=3.06 \pm 0.05 \mathrm{pb}$. An additional comparison is performed to the SUPERCHIC2 generator prediction of $\sigma_{\gamma \gamma \rightarrow \mu^{+} \mu^{-}}^{\mathrm{SC} 2}=3.45 \pm 0.05 \mathrm{pb}$, in which the absorptive effects are included at the amplitude level [10]. The differential crosssection is displayed in Fig. 2b. The EPA predictions are in good agreement with the measured cross-sections after correction for absorptive effects. The dominant uncertainties in both exclusive dilepton analyses is the shape uncertainty, stemming from the fit to the acoplanarity distribution. With greater background reduction through the use of proton tagging, this uncertainty can be significantly reduced.

A search for exclusive $W^{+} W^{-}$production at $\sqrt{s}=8 \mathrm{TeV}$ provided $3.0 \sigma$ evidence for Standard Model production relative to a background-only hypothesis [11]. The analysis was performed using the $e^{+} \mu^{-}$and $e^{-} \mu^{+}$visible final state to reject Drell-Yan and exclusive dilepton production. The same exclusivity requirements as those in the $\sqrt{s}=13 \mathrm{TeV}$ dilepton analysis are used and sig- 
nal events are triggered with the single lepton and different-flavour dilepton triggers. The invariant mass and the transverse momentum of the dilepton system are required to satisfy $m_{e \mu}>20 \mathrm{GeV}$ and $p_{T}>30 \mathrm{GeV}$ to remove background contamination. It is possible to select a region, $p_{T}>120$ $\mathrm{GeV}$, that is sensitive to anomalous quartic gauge couplings (aQGCs) while suppressing Standard Model contributions [12]. In this region, no significant excess is observed.

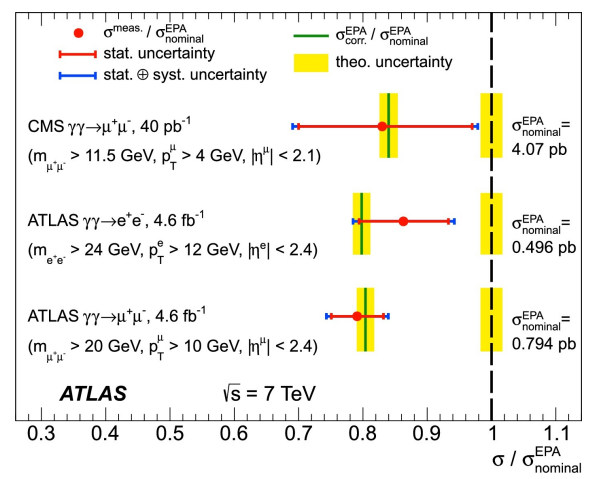

(a)

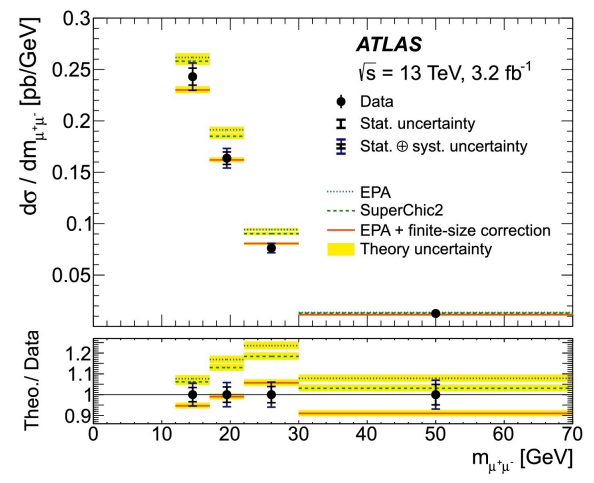

(b)

Figure 2: (a) Ratio of measured fiducial cross-section (red points) and QED prediction corrected for absorptive effects through the finite size approximation (green line) to uncorrected predictions (black line). A comparable result from the CMS Collaboration [13] is displayed [5]. (b) Differential cross-section for exclusive di-muon production as a function of the di-muon invariant mass [6]

\section{Diffractive Dijets}

Using a low pile-up data set with $\mu \sim 0.12$ and $\sqrt{s}=7 \mathrm{TeV}$, a measurement was performed of the differential cross-section for dijet production as a function of variables which discriminate between diffractive and non-diffractive processes [14]. Events were required to have exactly one reconstructed primary vertex and at least two jets with $p_{T}>20 \mathrm{GeV}$. The cross-section as a function of the rapidity gap, $\Delta \eta^{F}$, which is defined as the larger of the two regions in $\eta$ between \pm 4.8 and the nearest reconstructed track or calorimeter cluster, is displayed in Fig. 3a. It can be seen that diffractive processes become dominant in the region $\Delta \eta^{F}>2$. The cross-section is additionally presented differentially as a function of $\tilde{\xi}$, the fractional energy loss of the proton, as calculated from detected calorimeter clusters: $\tilde{\xi}=\sum p_{T} e^{ \pm \eta} / \sqrt{s}$. This cross-section is presented in Fig. 3b, after a selection of $\Delta \eta^{F}>2.0$ is applied to enrich the diffractive contribution to the sample. The POMWIG generator [15] does not simulate absorptive effects and so the sample is normalised to the lowest $\tilde{\xi}$ bin to measure this effect, also referred to as the rapidity gap survival probability, $S^{2}$. It is measured that $S^{2}=0.16 \pm 0.04$ (stat.) \pm 0.08 (syst.).

\section{Prospects with AFP}

During the LHC shutdowns between 2015-2017, the ATLAS Forward Proton (AFP) detectors were installed at a distance approximately $210 \mathrm{~m}$ from the ATLAS interaction point [16]. Comprising the AFP stations are silicon tracking detectors and Cerenkov Time-Of-Flight (TOF) detectors. 


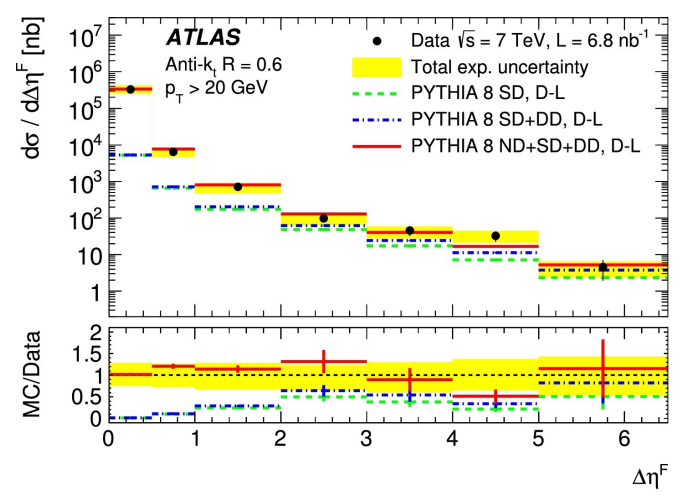

(a)

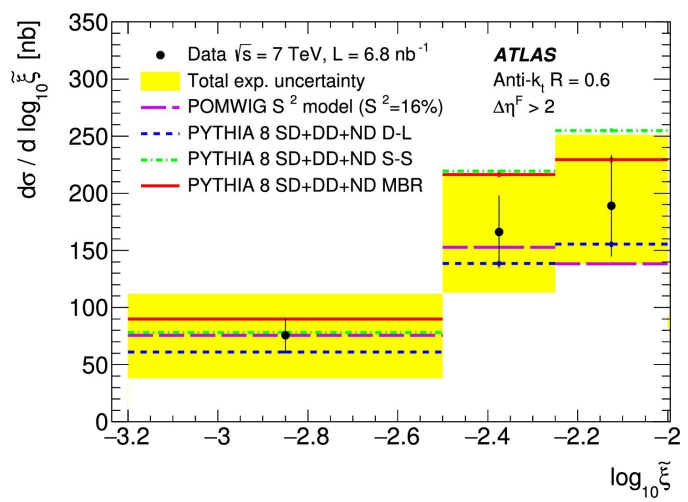

(b)

Figure 3: The differential dijet production cross-section as a function of (a) the larger of the two rapidity gaps from the calorimeter edges, (b) $\tilde{\xi}$ reconstructed from calorimeter clusters [14].

The primary physics goal of AFP is the identification and measurement of diffractively scattered protons that have been only slightly perturbed from the LHC beam. The TOF detectors are being commissioned at the time of writing, but with their projected time of arrival resolution of approximately $25 \mathrm{ps}$ it will be possible to identify the $z$-coordinate of the interaction point to a few $\mathrm{mm}$. This capability enriches the analysis potential of data collected under both low and high pile-up LHC running.

The first analysis performed using the AFP detectors is a proof of principle test to demonstrate

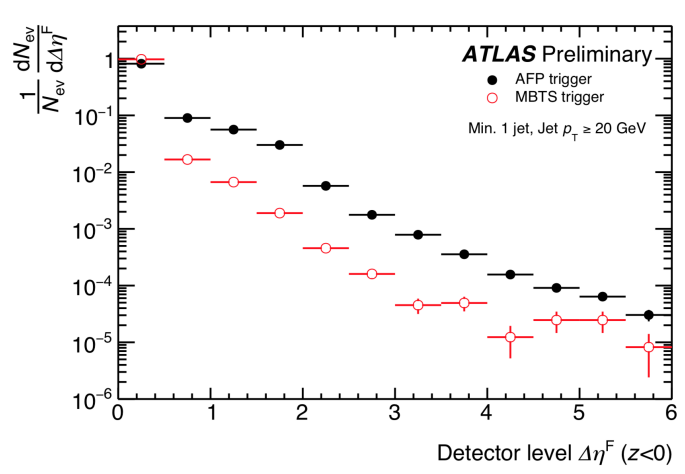

(a)

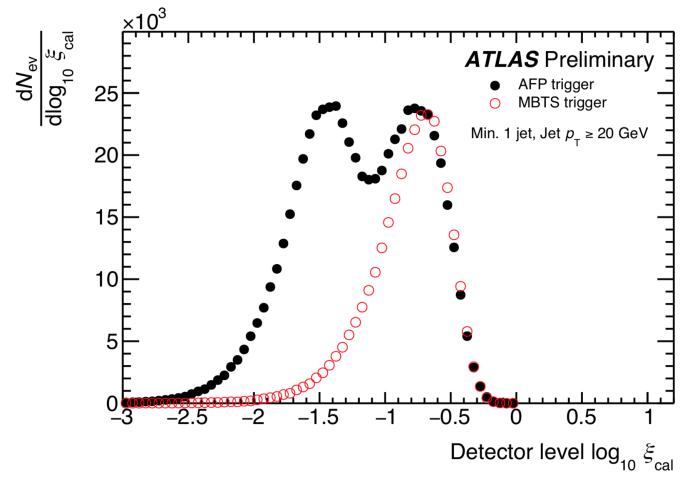

(b)

Figure 4: Comparison of AFP and minimum bias triggered samples (AFP trigger and MBTS trigger respectively). (a) $\Delta \eta^{F}$ measured from the calorimeter edge on the installed AFP side to the nearest track or cluster in $\eta$ with $p_{T}>200 \mathrm{MeV}$. (b) $\xi$ reconstructed from calorimeter clusters [17].

that AFP is capable of selecting a highly diffractive-enriched dijet sample. This analysis was performed at a low pile-up of $\mu \sim 0.3$ while only one side of AFP was installed [17]. Two separate data samples were selected during the same run: one triggered using an AFP trigger and one using a minimum bias trigger in the central ATLAS detector with coverage $2.08<|\eta|<3$.86. Additionally, both samples have the trigger requirement of at least one jet in the calorimeter with $p_{T}>10$ 
$\mathrm{GeV}$. Offline, it is required that all events have a single primary vertex with two associated tracks of $p_{T}>100 \mathrm{MeV}$ and that there is at least one jet in the calorimeter region $|\eta|<3.0$ with $p_{T}>20$ $\mathrm{GeV}$. An additional offline AFP requirement is applied to the AFP triggered sample to ensure that a good proton candidate was detected. Fig. 4a displays the normalised rapidity gap, $\Delta \eta^{F}$, spectrum for both samples, measured from the calorimeter edge on the side with the AFP detector to the nearest calorimeter cluster or track with $p_{T}>200 \mathrm{MeV}$. It can be seen that there is a greater fraction of events with large $\Delta \eta^{F}$ in the AFP triggered sample, indicating an enrichment in diffractive processes. The $\xi_{\text {cal }}$ distribution of each sample is compared in Fig. $4 \mathrm{~b}$, where $\xi_{\text {cal }}$ is the same observable as the previously described $\tilde{\xi}$,calculated from calorimeter clusters with $p_{T}>200 \mathrm{MeV}$. A double peak structure is observed in the AFP triggered sample, corresponding to diffractive processes at low $\xi_{\text {cal }}$ and non-diffractive processes at high $\xi_{\text {cal }}$. The non-diffractive events in the AFP sample are predominantly due to pile-up of non-diffractive events with processes that produce a very forward proton.

\section{Conclusion}

In proton-proton collisions provided by the LHC, ATLAS has performed cross-section measurements of exclusive dilepton production at $\sqrt{s}=7$ and $13 \mathrm{TeV}$, with measurements being consistent with predictions using the equivalent photon approximation and finite size approximation. At $\sqrt{s}=8 \mathrm{TeV}, 3 \sigma$ evidence is observed for the Standard Model exclusive production of $W^{+} W^{-}$pairs. Differential cross-sections of dijet production have been presented. From these, the rapidity gap survival probability is estimated to be $0.16 \pm 0.04$ (stat.) \pm 0.08 (syst.). It has been demonstrated that the new diffractive-specialised detectors, AFP, enable the selection of a highly diffractiveenriched sample. It should be possible to improve upon the analyses presented in this report using the proton-tagging capabilities of AFP.

\section{References}

[1] Landshoff, P. V. and Nachtmann, O, Zeitschrift fur Physik C35 (1987) 405.

[2] Chen, M. et al., Phys. Rev. D 7 (1973) 3485.

[3] ATLAS Collaboration, JINST 3 (2008) S08003.

[4] Budnev, V. M. et al., Nuclear Physics B 63 (1973) 519.

[5] ATLAS Collaboration, Phys. Lett. B749 (2015) 242 [1506.07098 [hep-ex] ].

[6] ATLAS Collaboration, Phys. Lett. B777 (2018) 303 [1708.04053 [hep-ex] ].

[7] Bahr, M. et al., Eur. Phys. J. C58 (2008) 639 [0803.0883 [hep-ph] ].

[8] Vermaseren, J. A. M., Nuclear Physics B 229 (1983) 347.

[9] Dyndal, M. and Schoeffel, L., Phys. Lett. B741 (2015) 66 [1410.2983 [hep-ph] ].

[10] Harland-Lang, L. A., Khoze, V. A. and Ryskin, M. G., Eur. Phys. J. C76 (2016) 9 [1508 . 02718 [hep-ph] ].

[11] ATLAS Collaboration, Phys. Rev. D94 (2016) 032011 [1607.03745 [hep-ex]].

[12] Chapon, E., Royon, C. and Kepka, O., Phys. Rev. D81 (2010) 074003 [0912. 5161 [hep-ph] ].

[13] CMS Collaboration, JHEP 01 (2012) 052 [1111.5536 [hep-ex] ].

[14] ATLAS Collaboration, Phys. Lett. B754 (2016) 214 [1511.00502 [hep-ex]].

[15] Cox, B. E. and Forshaw, J. R., Comput. Phys. Commun. 144 (2002) 104 [hep-ph/ 0010303 ].

[16] ATLAS Collaboration, Tech. Rep. CERN-LHCC-2015-009. ATLAS-TDR-024, May, 2015.

[17] ATLAS Collaboration, Tech. Rep. ATL-PHYS-PUB-2017-012, CERN, Geneva, Jul, 2017. 